\title{
Wide field of view optical coherence tomography for structural and functional diagnoses in dentistry
}

\author{
Joo Beom Eom \\ Jae Sung Ahn \\ Jonghyun Eom \\ Anjin Park
}




\title{
Wide field of view optical coherence tomography for structural and functional diagnoses in dentistry
}

\author{
Joo Beom Eom, Jae Sung Ahn, Jonghyun Eom, and Anjin Park* \\ Korea Photonics Technology Institute, Medical Photonics Research Center, Gwangju, Republic of Korea
}

\begin{abstract}
We report herein the fabrication and performance response of a three-dimensional (3-D) intraoral scan probe based on optical coherence tomography (OCT) that enables 3-D structural and functional diagnoses of the human teeth. The OCT system was configured using a swept-source OCT (SS-OCT) with a center wavelength of $1310 \mathrm{~nm}$. The scan probe was built using an MEMS mirror and an optical collimator. The implemented SS-OCT equipped with the MEMS-based scan probe yielded an axial resolution of $10 \mu \mathrm{m}$ and a scan range of $8 \times 8 \mathrm{~mm}^{2}$. Two-dimensional (2-D) cross-sectional images of the teeth were acquired by the scan probe based on the OCT. The 3-D volume image was acquired by combining a series of 2-D images, which includes internal and structural information of the human teeth. To utilize the OCT system as an intraoral scanner, partially overlapped 3-D volume images were sequentially acquired and stitched. The 3-D stitching was implemented based on an iterative closest point algorithm. The feasibility of the intraoral scan probe is demonstrated based on its ability to image and characterize the structure and function of the human teeth. () 2018 Society of Photo-Optical Instrumentation Engineers (SPIE) [DOI: 10.1117/1.JBO.23.7.076008]
\end{abstract}

Keywords: intraoral scanner; optical coherence tomography; dentistry; three-dimensional stitching.

Paper 180170R received Mar. 21, 2018; accepted for publication Jun. 26, 2018; published online Jul. 14, 2018.

\section{Introduction}

Currently, the gold standard for dental diagnosis is x-rays; however, the x-rays have limitations in resolution and contrast, making it difficult to diagnose microcracks in the teeth and early cavities. To solve these problems in dentistry, many researchers have studied optical coherence tomography (OCT) with high resolution and contrast. ${ }^{1-8}$ The studies continue to focus primarily on early diagnosis of caries, assessment of the quality and thickness of dentin, and restoration assessment and their monitoring. ${ }^{9}$ Even though OCT can provide high-resolution diagnosis images and volume images, the limitation of image acquisition rate and visual field of view (FOV) of the OCT system made it difficult to continuously acquire three-dimensional (3-D) structures or diagnostic images of the entire tooth in the oral cavity.

To obtain high-speed 3-D images in dentistry, 3-D intraoral scanners have been studied using various optical methods..$^{10}$ The 3-D intraoral scanners have been pursued to take digital impressions. ${ }^{11-16}$ The purpose of the impression-taking in dentistry is to transfer 3-D information from the patient's mouth to a model as accurately and comfortably as possible. ${ }^{17}$ The 3-D information has been utilized for achieving an accurate restoration or establishing the diagnosis and treatment plan for the patient. ${ }^{14-16}$ Most 3-D intraoral scanner, however, obtained only 3-D surface (structural) information from the teeth and precluded collection of diagnostic information from the oral cavity.

To extend the limited FOV, a 3-D stitching algorithm based on an iterative closest point (ICP) approach can be utilized to produce a composite 3-D volume images over a larger FOV from partially overlapped OCT volume images. The 3-D stitching algorithm consists mainly of two steps; feature points are extracted from each volume image, and one of the volumes is transformed to be merged to the other volume based on the feature points. Little attention has been paid to extend the limited FOV. Li et al. ${ }^{18}$ used blood vessel ridges, extracted from two-dimensional (2-D) OCT fundus images obtained by summing the OCT intensity along axial scan data (A-scans), as the feature points, and estimated a transformation based on the feature points. The method ${ }^{18}$ was the first work on the 3-D stitching for OCT volume images. Wang et al. ${ }^{19}$ used canny edge detector to extract the feature points and estimated the transformation based on the feature points using an ICP approach.

However, the methods mentioned above ${ }^{18,19}$ cannot be used for dental OCT volume images. The method based on the blood vessel ridges ${ }^{18}$ is limited to cases with clearly visible vascular structure. As the vascular structure is not visible in the cases of the dental OCT images, the method ${ }^{18}$ was not suitable for applying to the dental OCT volume images. The method based on the edge information ${ }^{19}$ sometimes fail to identify correct corresponding features between two OCT volume images due to changes in illumination and reflectivity unrelated to retinal characteristics, as well as speckle noise. ${ }^{20}$

In this study, we report the implementation of a handheld probe based on high-speed OCT that can acquire surface (structural) as well as diagnostic (functional) information of the teeth and gums. The proposed handheld probe used a highspeed swept-source OCT (SS-OCT) system equipped with an MEMS-based scan probe to acquire 3-D volume images that contain both surface and diagnostic information, as reconstructed from a series of 2-D cross-sectional images. To extend the FOV, we extracted the feature points, suitable for the dental 
OCT volume images, by thresholding each volume image, and then implemented an ICP approach, which produces a composite 3-D volume image over a large FOV from partially overlapped volume images. In the experiments, we demonstrated the feasibility of the handheld probe and confirmed that the OCT-based handheld probe could be utilized for structural and functional diagnoses in dentistry.

\section{Experimental Setup for Handheld Probe Based on Optical Coherence Tomography}

Figure 1 shows the SS-OCT system, which was constructed based on a Mach-Zehnder interferometer. The system is equipped with an MEMS-based scan probe to be used inside the mouth. The system comprises five modules: a wavelength swept source, an optical fiber interferometer, an MEMS mirror, a controller, and a digitizer, used for acquiring and displaying OCT volume images. As the fast scanning speed and enhanced light penetration to the deeper layer were required to utilize the OCT system as an intraoral scan probe, a wavelength swept laser source (Thorlabs, SL1310V1-20048), with a central wavelength of $1310 \mathrm{~nm}$, a scanning rate of $200 \mathrm{kHz}$, and a full-width-at-halfmaximum bandwidth of $100 \mathrm{~nm}$, is used as the light source. ${ }^{15}$ The laser beam from the swept source is guided to the optical fiber Mach-Zehnder interferometer as shown in Fig. 1, which is divided into a sample arm and reference arm through a 90:10 fiber coupler (Thorlabs, TW1300R2A2). Figure 2 shows an experimental setup, which sequentially acquires the volume images. The handheld probe was fixed, and a stage [Fig. 2(a)] was moved after acquiring a volume image.

The MEMS-based scan probe was fabricated to act as a sample arm. The beam reflected from the reference armconsisting of a mirror, collimator (Thorlabs, F220APC-1310), lens (Thorlabs, AC127-030-C-ML), a polarization controller (Thorlabs, PLC-900), and iris (Thorlabs, SM05D5D)—was utilized as the reference beam of the system. Herein, the role of the polarization controller was to maintain the polarization states of two arms for excellent visibility of interference, and the role of the iris was to adjust the optical power at the reference arm appropriately. The lights reflected from the sample and the reference arm interfered with each other through a 50:50 coupler (Thorlabs, TW1300R5A2), and the interference signal was detected by a 1.6-GHz fiber-coupled balanced amplified photodiode (Thorlabs, PDB480C-AC). The photocurrent, i.e., the spectral interference signal, was converted into a voltage and directed to a 12-bit high-speed digitizer (Gage, CES123G2).

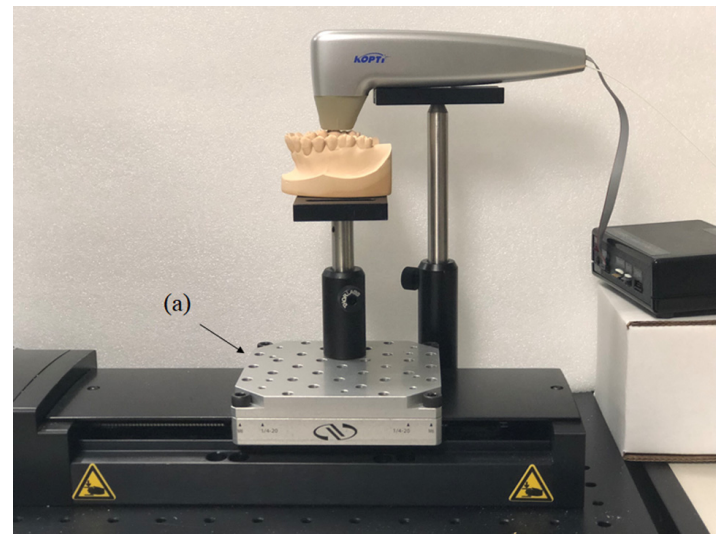

Fig. 2 Experimental setups for sequentially acquiring OCT volume images.

The spectrum interpolation for resampling $k$-space was performed on all A-scans before the supplication of the fast Fourier transform. ${ }^{21}$

Figure 3 shows a schematic drawing of the MEMS-based handheld probe [Fig. 3(a)] and photographs of an implemented probe [Figs. 3(b)-3(d)]. The size of the probe is $163 \mathrm{~mm}$ $163 \mathrm{~mm} \times 58 \times 33 \mathrm{~mm}$, as shown in Figs. 3(b)-3(d). An optical fiber patchcord (FC/APC type connector) was connected with the collimator (Thorlabs, F220APC-1310) to establish the collimation beam. The collimation beam was launched at a scan lens (Thorlabs, AC254-030-C) through the MEMS mirror [Mirrorcle Technologies. Inc., A4SR8.1-4200AL. a mirror diameter: $4.2 \mathrm{~mm}$, tilt angle $(\max ): \pm 5 \mathrm{deg}$, actuator board size: $8 \mathrm{~mm} \times 8 \mathrm{~mm}$ ). To achieve a vertical collimated beam, a jig was designed and fabricated. The scan lens used herein was an achromatic doublet-lens with a diameter of $1 \mathrm{in}$. and a focal length of $30 \mathrm{~mm}$. Table 1 summarized the individual components, optical elements and manufacturers, used for the intraoral scan probe.

Figure 4 shows examples of 2-D cross-sectional images [Fig. 4(a)] and a 3-D volume image [Fig. 4(b)], which were acquired by the OCT system equipped with the MEMSbased probe. The scan range was $\sim 8 \times 8 \mathrm{~mm}^{2}$. The number of A-scans samples was 1024, and the 2-D cross-sectional image was constructed using 256 A-scans, whereas the 3-D volume images comprised 256 cross-sectional images. In other words, the size of the volume acquired by the OCT system was $1024($ A-scan $) \times 256($ B-scan $) \times 256($ C-scan $)$. The axial

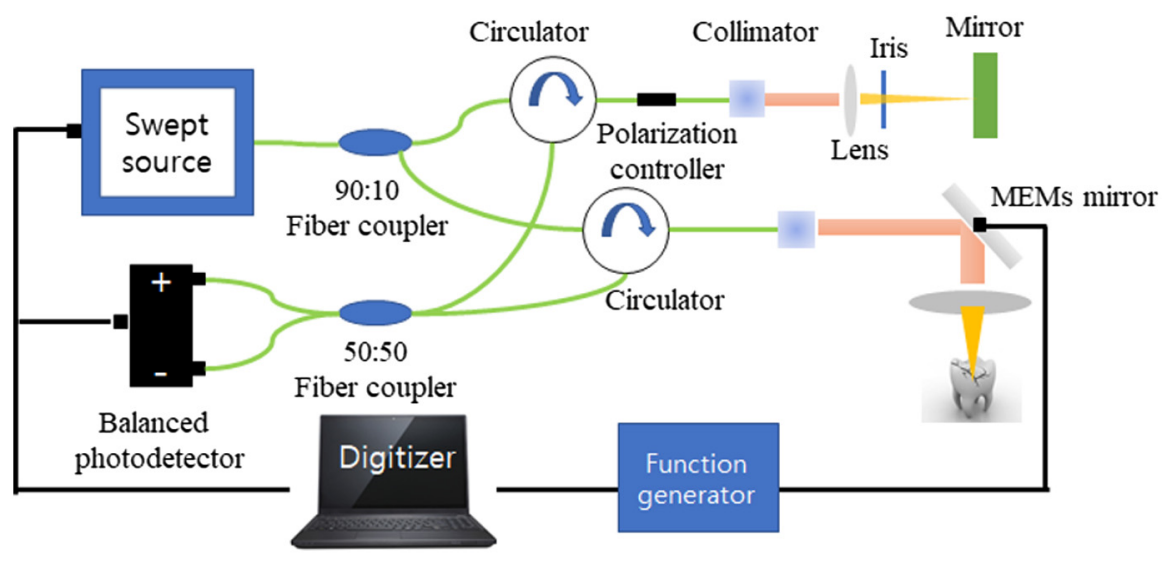

Fig. 1 Schematic of SS-OCT system with MEMS-based scan probe for use as an intraoral scanner. 


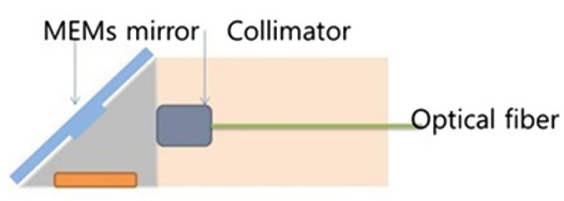

Lens

(a)

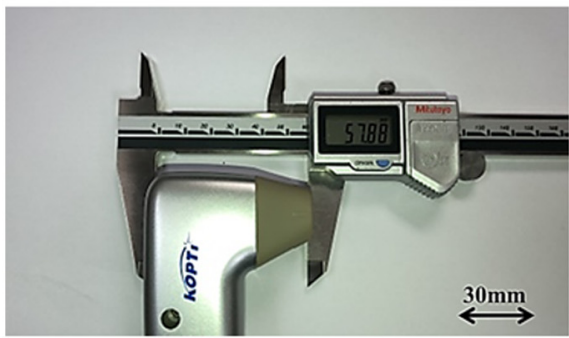

(c)

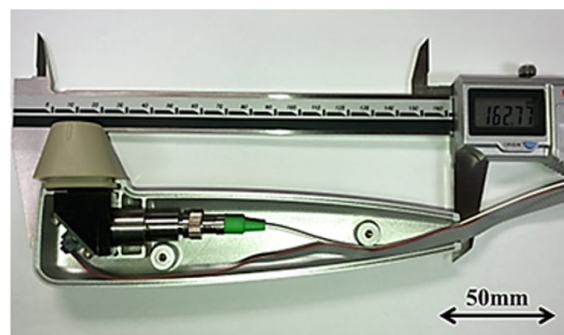

(b)

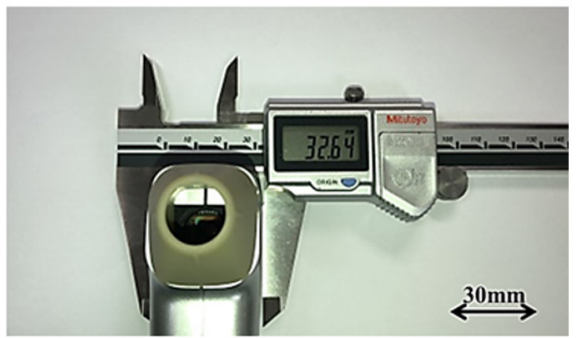

(d)

Fig. 3 Schematic of (a) MEMS-based scan probe and (b-d) its photographs.

Table 1 Individual components, manufacturers, and optical elements, used for intraoral scan probe.

\begin{tabular}{lll} 
Component & \multicolumn{1}{c}{ Manufacturer } & \multicolumn{1}{c}{ Optical elements } \\
\hline Collimator & Thorlabs, F220APC-1310 & $\begin{array}{l}\text { Size of the incident } \\
\text { beam on the lens: } 2 \mathrm{~mm}\end{array}$ \\
Scan lens & Thorlabs, AC254-030-C & $\begin{array}{l}\text { Working distance: } \\
30 \mathrm{~mm}\end{array}$ \\
& & \\
MEMS mirror & Mirrorcle Technologies, & $\begin{array}{l}\text { Mirror diameter: } \\
\text { Inc., A4SR8.1-4200AL }\end{array}$ \\
& & $4.2 \mathrm{~mm}$, tilt angle (max), \\
\pm 5 deg actuator board \\
& size: $8 \mathrm{~mm} \times 8 \mathrm{~mm}$ \\
\hline
\end{tabular}

resolution of the system was measured to be $10 \mu \mathrm{m}$ in air. The lateral resolution ${ }^{22}$ was measured to be $25 \mu \mathrm{m}$ in the air, where the central wavelength was $1310 \mathrm{~nm}$, the focal length was $30 \mathrm{~mm}$, and the size of the incident beam on the objective lens was $2 \mathrm{~mm}$. Using a swept laser with a swept source at $200 \mathrm{kHz}$, the frame rate of 2-D cross-sectional images was $~ 780 \mathrm{fps}$, and one 3-D volume image was acquired within $0.35 \mathrm{~s}$.

\section{Three-Dimensional Stitching Algorithm for Dental Volume Images}

The goal of the intraoral scanner was to digitally impress the specific areas of the teeth and gums that the dentists will treat. To scan the areas-whose dimensions are beyond the FOV of the MEMS-based probe - the proposed scanner merges partially overlapped volume images acquired by the OCT system. Figure 5 shows an example of the partially overlapped 3-D volume images [Figs. 5(b)-5(g)] obtained from the oral teeth model [Fig. 5(a)].

The process of merging the partially overlapped 3-D volumes is known as 3-D stitching. The stitching of 3-D volume images consists of two steps: (1) estimating a transformation, composed of a translation vector and a rotation matrix, of $i+1$ 'th OCT volume from $i$ 'th OCT volume, and (2) transforming the $i+1$ 'th OCT volume into the $i$ 'th OCT volume based on the estimated transformation.

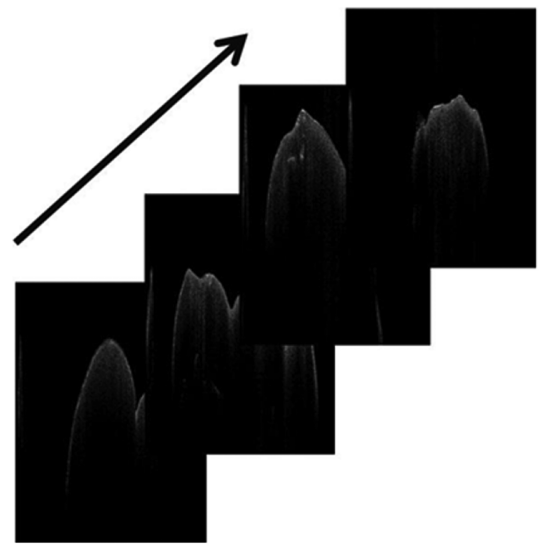

(a)

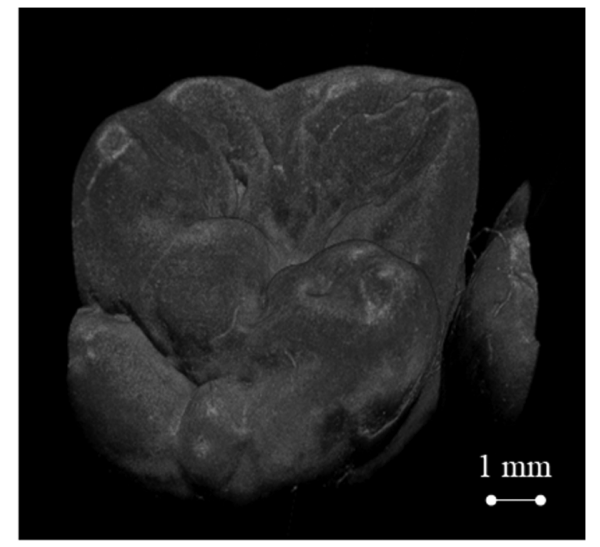

(b)

Fig. 4 (a) 2-D cross-sectional images and (b) 3-D volume image of human tooth acquired by the implemented OCT system. 


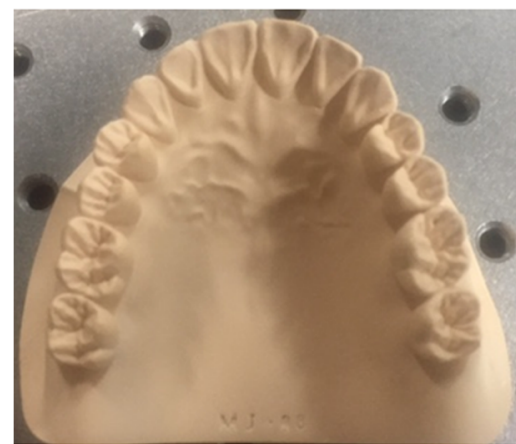

(a)

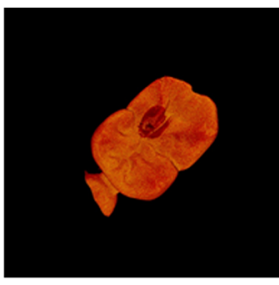

(b)

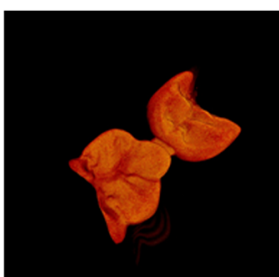

(e)

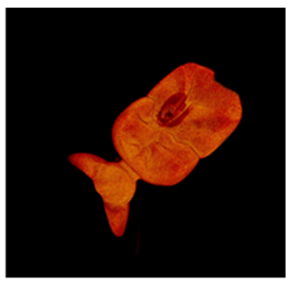

(c)

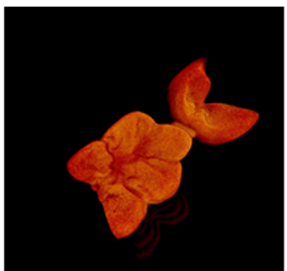

(f)

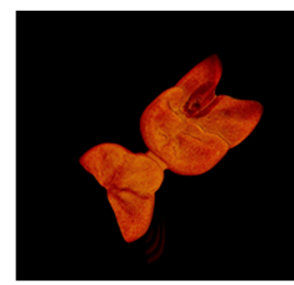

(d)

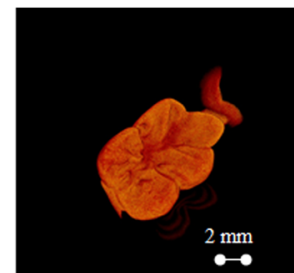

(g)

Fig. 5 Examples of 3-D volume images acquired by the OCT system: (a) oral teeth model and (b)(g) sequentially acquired 3-D volume images.

The transformation between two volume images was estimated based on an ICP approach. ${ }^{23}$ First, the point cloud was extracted as the feature points from each volume image. As the enamel layer in the dental OCT images has higher intensity values than other dental tissues, this paper uses voxels corresponding to the enamel layer as feature points. To extract the enamel layer from the dental OCT volume images, which has relatively higher value than those in other regions of the teeth, we used the thresholding method. Herein, the set of coordinates of voxels that have higher intensities than a predefined threshold was collected as the point cloud. Figure 6 shows point clouds extracted from the 3-D volume images of Figs. 5(b)-5(g). The threshold value used to extract the point clouds shown in Fig. 6 was 75.
The ICP algorithm then estimates a rotation matrix $\mathbf{R}^{i+1}$ and a translation vector $\mathbf{t}^{i+1}$ between the two point clouds $\mathbf{P}^{i}$ and $\mathbf{P}^{i+1}$, so that $\mathbf{P}^{i+1}$ is transformed to optimally match $\mathbf{P}^{i}$. Figure 7 shows typical transformation outcomes of the point cloud of Fig. 6(b) in consecutive iterations, based on Fig. 6(a), whereas Fig. 7(d) shows the result of the final transformed point cloud of Fig. 6(b) that best matches the point cloud of Fig. 6(a).

The ICP algorithm was used to calculate the rotation matrix $\mathbf{R}^{i+1}$ and translation vector $\mathbf{t}^{i+1}$ to obtain an optimum match of the OCT volume images - acquired in the current frame- to the OCT volume acquired in the previous frame. The $\mathbf{R}^{i+1}$ and $\mathbf{t}^{i+1}$ calculated using the ICP algorithm were then used to transform the $(i+1)^{\prime}$ th acquired OCT volume $\mathbf{V}^{i+1}$, based on the $i$ 'th acquired OCT volume $\mathbf{V}^{i+1}$, as follows:

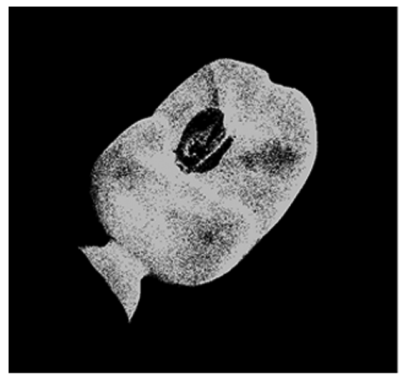

(a)

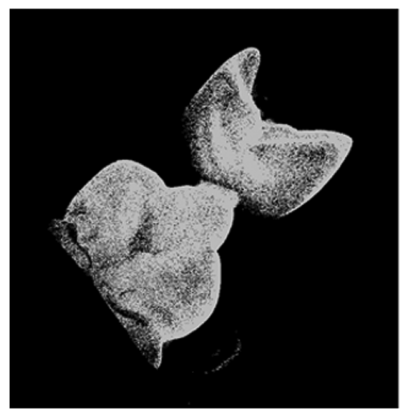

(d)

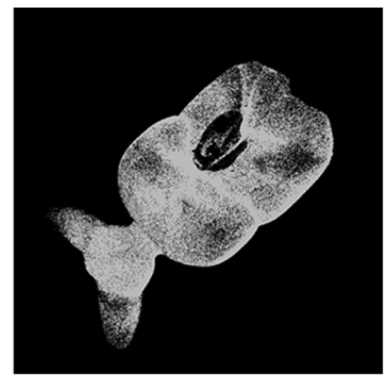

(b)

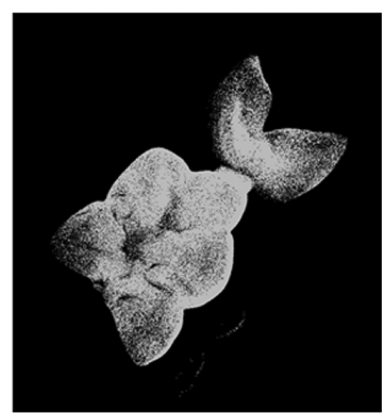

(e)

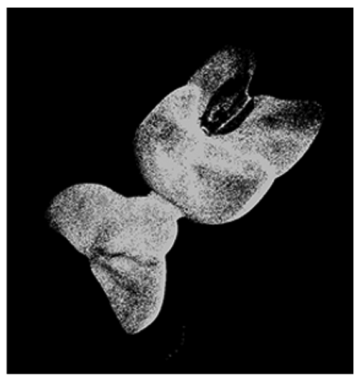

(c)

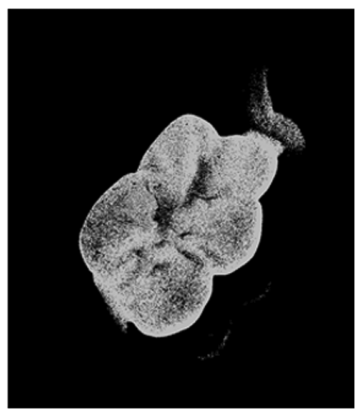

(f)

Fig. 6 Example of point clouds extracted from the volume images of Fig. 5(b) (a) point cloud extracted from Fig. 5(b), (b) point cloud extracted from Fig. 5(c), (c) point cloud extracted from Fig. 5(d), (d) point cloud extracted from Fig. 5(e), (e) point cloud extracted from Fig. $5(\mathrm{f})$, and (f) point cloud extracted from Fig. 5(g). 


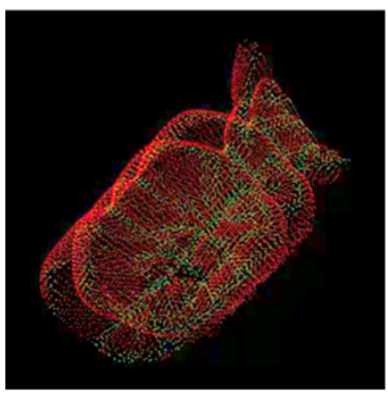

(a)

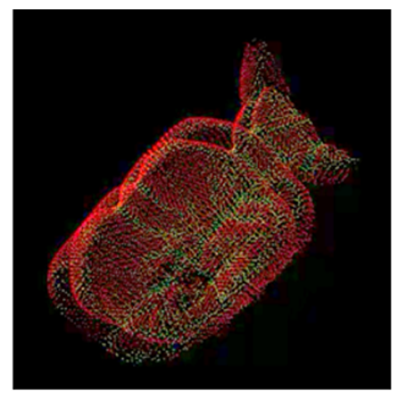

(b)

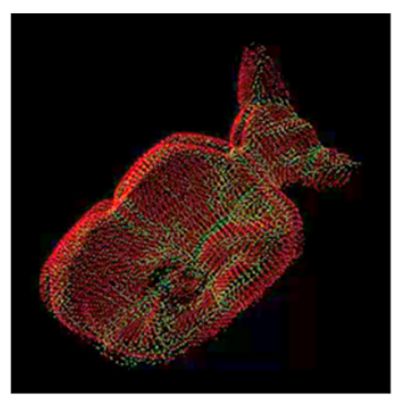

(c)

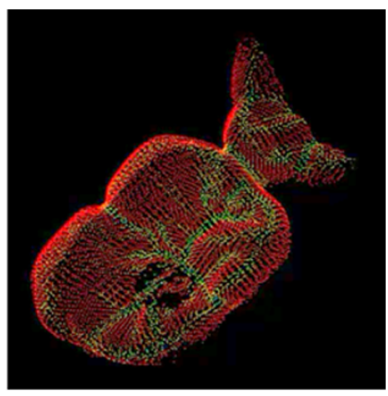

(d)

Fig. 7 Transformation outcomes of the point cloud of Fig. $6(\mathrm{~b})$ based on the point cloud of Fig. $6(\mathrm{a})$ in (b) the first iteration, (c) second iteration, and (d) third iteration from (a) the initial position of the point clouds.

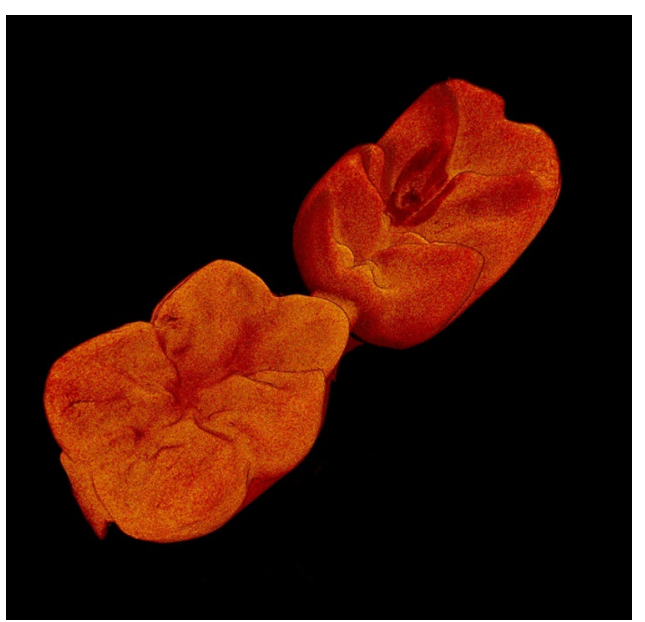

Fig. 8 3-D stitching result of partially overlapped OCT volumes of Figs. 5(b)-5(g).

$\boldsymbol{\nu}_{n}^{i+1}=\mathbf{R}^{i+1} \cdot \boldsymbol{\nu}_{n}^{i+1}+\mathbf{t}^{i+1}$,

where $\boldsymbol{\nu}_{n}^{i+1}$ denotes the $n^{\prime}$ th voxel of $\mathbf{V}^{i+1}$. In other words, each voxel of the $\mathbf{V}^{i+1}$ is transformed to a corresponding voxel in $\mathbf{V}^{i}$. Figure 8 shows the result of 3-D stitching of the partially overlapped volumes, whereby the volumes of Figs. 5(b)-5(g) are used as an input for the result.

\section{Results and Discussion}

The proposed handheld probe, composed of OCT volume image acquisition and 3-D stitching algorithm, was performed on a workstation computer with a single Intel Xeon E5-2609 v2 processor. The computer hosts the digitizer card as well as a CUDA (compute unified device architecture)-enabled NVIDIA GTX 1080 single GPU card. CUDA version 8.0, NVIDIA's parallel computing platform and programming model, was employed to program and harness the power of the GPU. The motherboard supports PCI-Express 3.0, allowing maximum bandwidth for data transfer among the main memory of the computer, digitizer memory, and GPU memory.

To perform the volume image acquisition and 3-D stitching algorithm efficiently, we implemented a self-developed multithreaded C++ software that was built and compiled in Microsoft Visual Studio 2013. Figure 9 shows the flow of the implemented software. Once the new volume image was acquired, the 3-D stitching algorithm converted the volume image into the global coordinate system based on the previous volume images. The volume image acquisition step immediately acquired the next volume without waiting for the complete the 3-D stitching step. See Fig. 10 for the software implemented handheld probe.

To confirm the applicability of the proposed handheld probe based on the OCT system equipped with an MEMSbased intraoral scan probe, the OCT volume images of the extracted human teeth were sequentially acquired, as shown in Figs. 11(a)-11(d). The overlapping percentage of the sequentially acquired OCT volumes was $>70 \%$ in the conducted experiments. Figures 11(e)-11(h) show point clouds extracted from the OCT volumes of Figs. 11(a)-11(d). As shown in Figs. 11(e)-11(h), voxels located at the surface (the hard enamel layer) of the teeth were extracted as the point cloud as the voxel intensities at the surface had relatively higher values than those in other regions of the teeth. Therefore, the set of coordinates of

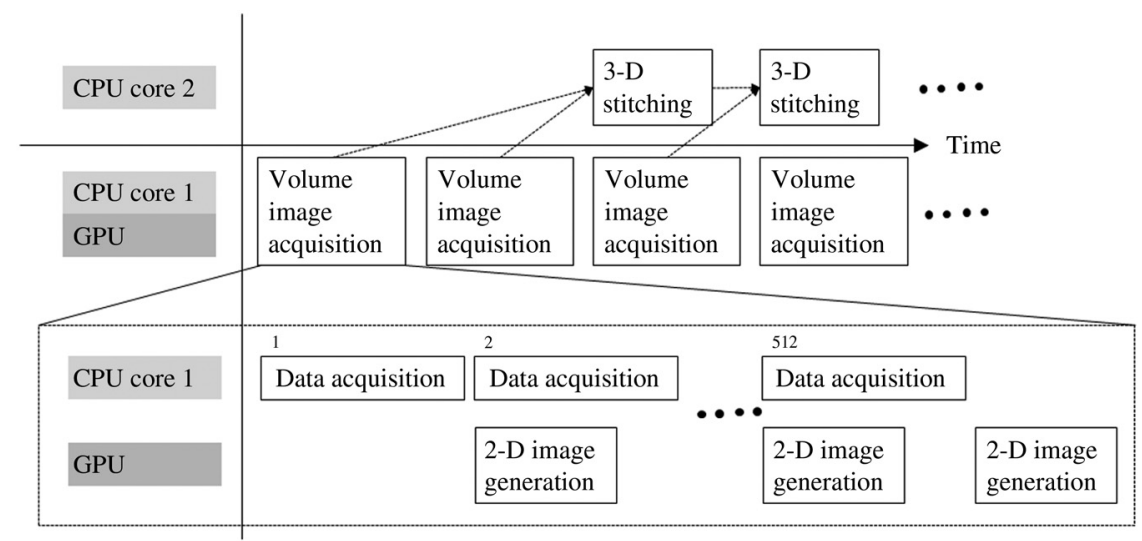

Fig. 9 Flowchart of self-developed multithreaded software. 


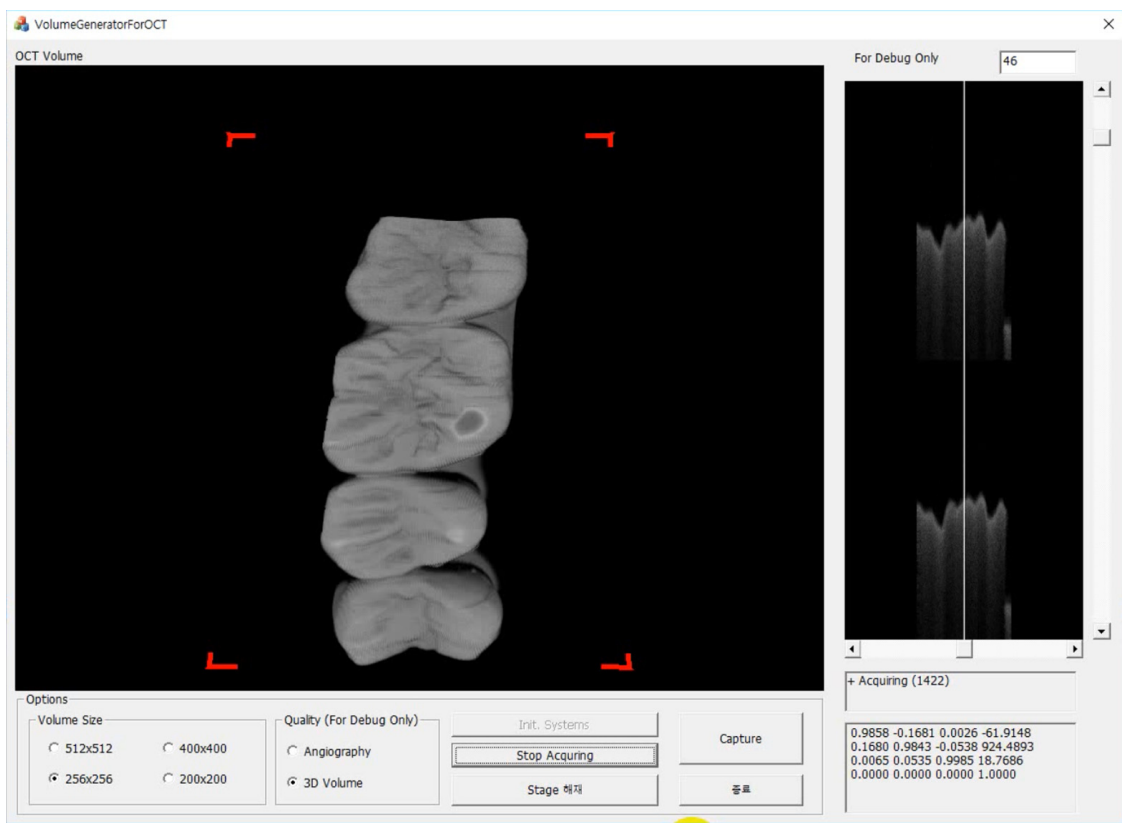

Fig. 10 Example of a multimedia still image (Video 1, MPEG4, 1.12 MB [URL: https://doi.org/10.1117/1 .JBO.23.7.076008.1]).

the voxels having a higher intensity than a predefined threshold was considered as the point cloud.

The point clouds were then used to estimate the rotation matrix and translation vector between two adjacent volume images using the 3-D stitching algorithm. The algorithm was implemented based on the point cloud library. ${ }^{24}$ The average processing time for pairwise stitching was $\sim 0.3 \mathrm{~s}$ on the workstation computer. Figures 11(i)-11(k) show 3-D stitching results for Figs. 11(a)-11(d), based on the rotation matrix and translation vector. Figure 11(i) shows the outcome volume when the volume of Fig. 11(b) is transformed into the volume of Fig. 11(a). Correspondingly, Fig. 11(j) shows the volume outcome when the volume of Fig. 11(c) is transformed into the volume of Fig. 11(b), whereas Fig. 11(k) shows the outcome when the volume of Fig. 11(d) is transformed into the volume of Fig. 11(c).

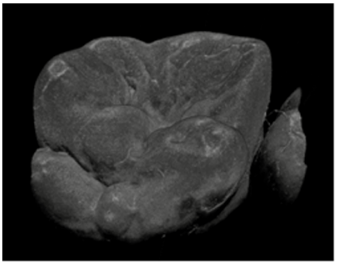

(a)

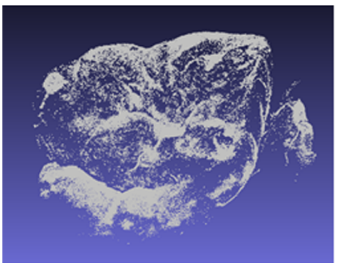

(e)

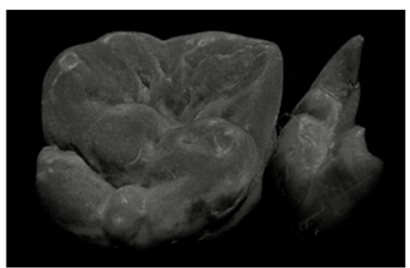

(i)

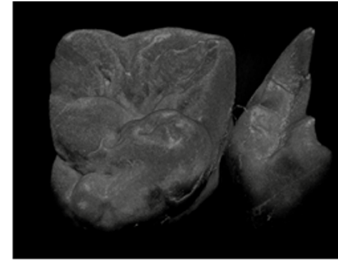

(b)

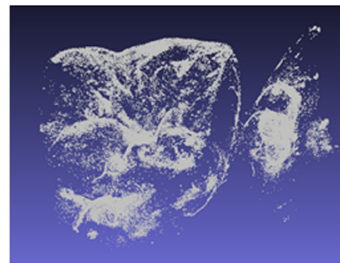

(f)

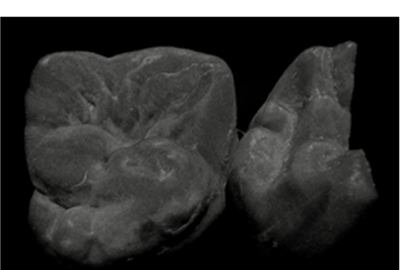

(j)

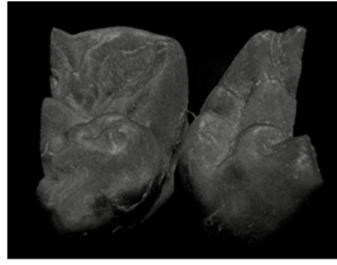

(c)

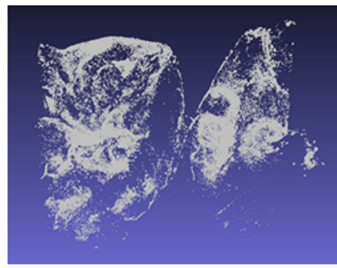

(g)

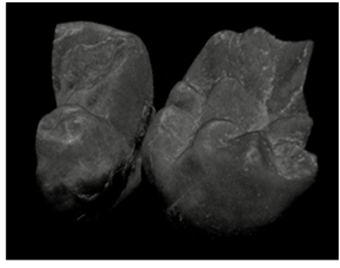

(d)

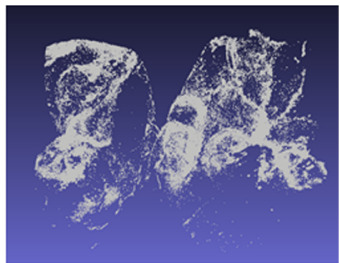

(h)

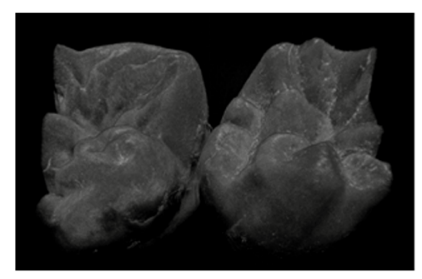

(k)

Fig. 11 Stitching results for partially overlapped OCT volumes: (a-d) sequentially acquired OCT volumes, (e-h) corresponding point clouds, and (i-k) results of 3-D stitching of two adjacent volumes. 


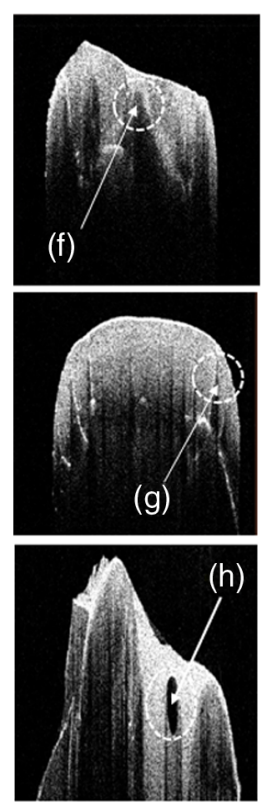

(a)
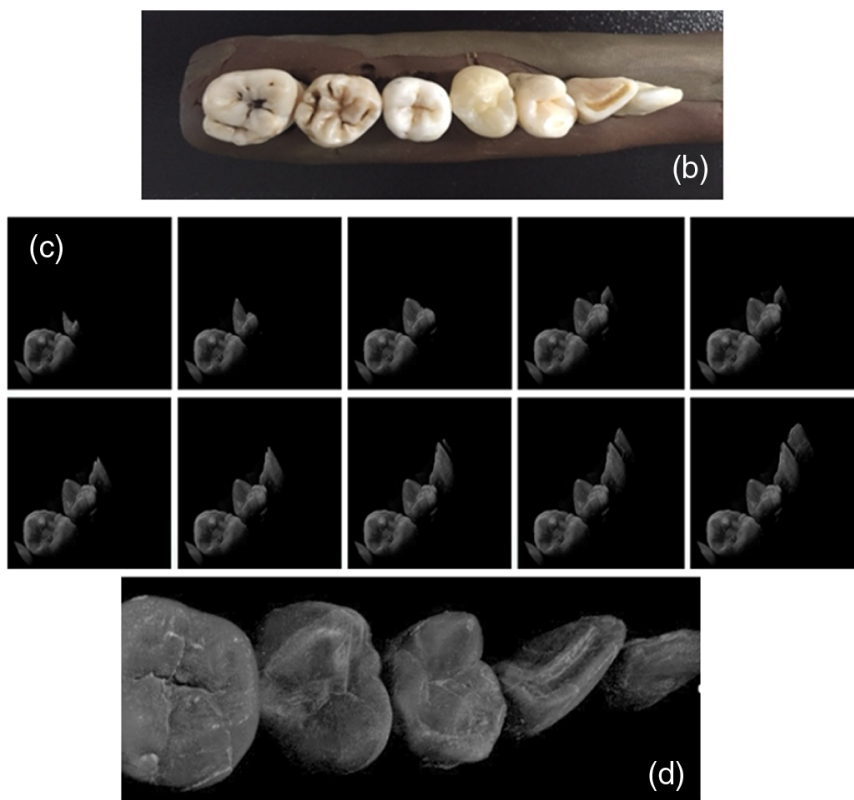

(d)

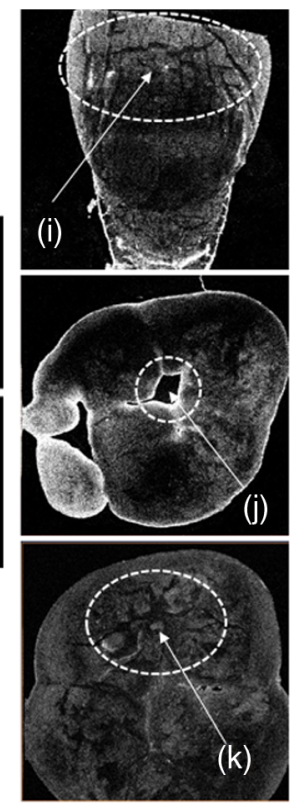

(e)

Fig. 12 Results of stitching extracted human teeth acquired by implementing a handheld probe based on OCT: (a) 2-D cross-sectional images in the $X Y$-plane, (b) photography of extracted human teeth, (c) stitching result for 10 sequentially acquired volumes, (d) final result of the intraoral scanner, (e) 2-D cross-sectional images in the $X Z$-plane, (f, g) internal cracks, (h) imperfections of resin restoration, (i-k) external cracks, and $(\mathrm{j}, \mathrm{k})$ caries lesions.

Figure 12(c) shows an example of stitching of OCT volumes that were successively acquired from extracted human teeth [Fig. 12(b)] and Fig. 12(d) is a 3-D image of the enlarged tooth of Fig. 12(b). The image of the stitched 3-D volume contains 2-D cross-sectional information of all parts of the teeth. Figure 12(a) shows 2-D images reconstructed in the $X Y$-plane, whereas Fig. 12(e) shows 2-D images reconstructed in the $X Z$ plane. As it can be seen from the 2-D cross-sectional images, the presence of crack [Figs. 12(f), 12(g), and 12(i)], tooth decay [Figs. 12(i) and 12(k)], and teeth resin treatment [Fig. 12(h)] can be confirmed.

In the case of a conventional intraoral scanner, rapid acquisition of the 3-D structure of the tooth has made it possible to manufacture a dental prosthesis. However, such an intraoral scanner has information about the surface of the tooth, which is impossible to diagnose the teeth or the oral cavity. However, the proposed technique has not only a 3-D structure image of the tooth, but also a tomographic image of the inside of the tooth and the oral cavity. In this way, the proposed handheld probe based on the OCT can help the dentists to diagnose the tooth as well as diseases in the oral cavity, such as oral cancer. It is expected to be extensively used in future diagnoses and teeth treatments.

However, imaging systems using optical methods are not able to penetrate metals. The OCT system cannot penetrate metals as well. Therefore, the proposed handheld probe has a limitation that it can acquire a structural image but cannot acquire a diagnostic image of the tooth restored with a metal material, for example, an amalgam. In addition, motion artifacts can arise when the OCT volume images were acquired in vivo. In the experimental results, the motion artifacts were not introduced, as the scanning probe was fixed and the stage was moved after acquiring each volume image to acquire the next volume image, as shown in Fig. 2. If the OCT volume images can be acquired at more than $20-\mathrm{Hz}$ video rate, which results in motionfree imaging, the proposed handheld probe has excellent potential for use in structural and functional applications in dentistry. As high-quality video-rate OCT imaging system has recently received a lot of attention, ${ }^{25}$ we expect that the evolution of the OCT leads to a significant increase in the data acquisition speed and will bring the realization of high-speed OCT systems shortly.

\section{Conclusions}

We proposed and presented an intraoral handheld probe based on an OCT system. In this system, the optical patch cord was connected to the collimator, and the parallel light was incident on the scan lens through the MEMS mirror to produce the 3-D volume images. We implemented the SS-OCT system with a scanning rate of $200 \mathrm{kHz}$ and multithreaded $\mathrm{C}++$ software to sequentially acquire 3-D volume images faster than the commercial OCT system. 3-D stitching algorithm, suitable for the dental OCT volume images, was also introduced based on the characteristic of the teeth in that the surface had relatively higher intensities than those in other regions of the teeth and gums to stitch the partially overlapped 3-D volume images. To confirm the function of the handheld probe, the OCT volume images were acquired from extracted human teeth. Image acquisition of the teeth using the OCT-based handheld probe yielded 3-D structural and diagnostic images. Based on the reconstructed 3-D scanned images of human teeth, we can infer that the proposed handheld probe has excellent potential for use in structural and functional characterization applications in dentistry.

\section{Disclosures}

The authors have no relevant financial interests in this article and no potential conflicts of interest to disclose. 


\section{Acknowledgments}

This research was a part of the project titled "Development of marine material based near infrared fluorophore complex and diagnostic imaging instruments (No. 20170263)" funded by the Ministry of Oceans and Fisheries, Korea. We would like to express our sincere appreciation to the members of the Department of Orthodontics at Chonnam National University Dentistry Hospital for advice on the provision of teeth and the resulting images.

\section{References}

1. K. Al-Azri et al., "Optical coherence tomography use in the diagnosis of enamel defects," J. Biomed. Opt. 21(3), 036004 (2016).

2. S. H. Lee et al., "Dental optical coherence tomography: new potential diagnostic system for cracked-tooth syndrome," Surg. Radiol. Anat. 38(1), 49-54 (2016).

3. V. Damodaran et al., "Optical coherence tomography based imaging of dental demineralisation and cavity restoration in $840 \mathrm{~nm}$ and $1310 \mathrm{~nm}$ wavelength," Opt. Lasers Eng. 83, 59-65 (2016).

4. A. Algami et al., "Enamel thickness determination by optical coherence tomography: in vitro validation," Caries Res. 50(4), 400-406 (2016).

5. S. H. Han et al., "Non-destructive evaluation of an internal adaptation of resin composite restoration with swept-source optical coherence tomography and micro-CT," Dent. Mater. 32, e1-e7 (2016).

6. C. S. Sampaio et al., "Effect of restorative system and thermal cycling on the tooth-restoration interface-OCT evaluation," Oper. Dent. 41(2), 162-170 (2016).

7. M. S. Segarara et al., "Three-dimensional analysis of enamel crack behavior using optical coherence tomography," J. Dent. Res. 96(3), 308-314 (2017).

8. T. Ueno et al., "Optical analysis of enamel and dentin caries in relation to mineral density using swept-source optical coherence tomography," J. Med. Imaging 3(3), 035507 (2016).

9. M. Machoy et al., "The use of optical coherence tomography in dental diagnostics: a state-of-the-art review," J. Healthc. Eng. 2017, 1-31 (2017).

10. A. J. Ireland et al., "3D surface imaging in dentistry-what we are looking at," Br. Dent. J. 205(7), 387-392 (2008).

11. S. Logozzo et al., "Recent advances in dental optics-Part I: 3D intraoral scanners for restorative dentistry," Opt. Lasers Eng. 54, 203-221 (2014).

12. N. S. Birmbaum et al., "3D digital scanners: a high-tech approach to more accurate dental impressions," Inside Dent. 5(4), 70-74 (2009).

13. F. Beuer et al., "Digital dentistry: an overview of recent developments for CAD/CAM generated restorations," Br. Dent. J. 204(9), 505-511 (2008).

14. M. Zimmermann et al., "Intraoral scanning systems-a current overview," Int. J. Comput. Dent. 18(2), 101-129 (2015).

15. F. Mangano et al., "Intraoral scanners in dentistry: a review of the current literature," BMC Oral Health 17(1), 149 (2017).

16. M. Imburgia et al., "Accuracy of four intraoral scanners in oral implantology: a comparative in vitro study," BMC Oral Health 17(1), 92 (2017).
17. L. Culp et al., "Digital technology in implant dentistry," in Dental Implant Prosthetics, 2nd ed., C. E. Misch, Ed., pp. 700-723, Mosby, Missouri (2015).

18. Y. Li et al., "Automatic montage of SD-OCT data sets," Opt. Express 19(27), 26239 (2011).

19. X. Wang et al., "An iterative closest point approach for the registration of volume human retina image data obtained by optical coherence tomography," Multimedia Tools Appl. 76(5), 6843-6857 (2017).

20. J. Zheng et al., "Salient feature region: a new method for retinal image registration," IEEE Trans. Inf. Technol. Biomed. 15(2), 221-232 (2010).

21. J. B. Eom et al., "Ball lens based lensed patch cord probes for optical coherence tomography in the field of dentistry," Opt. Commun. 285(21-22), 4333-4337 (2012).

22. J. G. Fujimoto and W. Drexler, Optical Coherence Tomography: Technology and Applications, 2nd ed., Springer International Publishing, Switzerland (2015).

23. P. J. Besl and N. D. McKay, "A method for registration of 3-D shapes," IEEE Trans. Pattern Anal. 14(2), 239-256 (1992).

24. D. Holz et al., "Registration with the point cloud-a modular framework for aligning in 3-D," IEEE Robot. Autom. Mag. 22(4), 110-124 (2015).

25. W. Wieser et al., "High definition live 3D-OCT in vivo: design and evaluation of a 4D OCT engine with $1 \mathrm{GVoxels} / \mathrm{s}$," Biomed. Opt. Express 5(9), 2963 (2014).

Joo Beom Eom is a principal researcher at Korea Photonics Technology Institute. He received his BS degree in physics from Yeungnam University in 2000, and his MS and PhD degrees in information and communications from Gwangju Institute Science and Technology in 2002 and 2011, respectively. He is the author of 18 journal papers and has more than 50 patents. His current research interests include optical coherence tomography, photoacoustic imaging, spectroscopy, and terahertz imaging and spectroscopy.

Jae Sung Ahn is a senior researcher at Korea Photonics Technology Institute. He received his $\mathrm{BS}, \mathrm{MS}$, and $\mathrm{PhD}$ degrees in physics from Seoul National University in 2006, 2008, and 2015, respectively. He is the author of 13 journal papers. His research interests are 3-D optical microscopy, scanning probe microscopy and fluorescence lifetime imaging microscopy.

Jonghyun Eom received his BS degree in biomedical engineering at Yonsei University, Republic of Korea, in 2008. He received his $\mathrm{MS}$ and $\mathrm{PhD}$ degrees in medical system engineering and in biomedical science and engineering at Gwangju Institute Science and Technology, in 2010 and 2017, respectively. Currently, he is a senior researcher at Korea Photonics Technology Institute. His research interests include photoacoustic imaging, optical coherence tomography, fluorescence imaging, and optical measurements.

Anjin Park is a principal researcher at Korea Photonics Technology Institute. He received his $\mathrm{PhD}$ in Digital Media Engineering from the Soongsil University, Republic of Korea, in 2009. His current research interests include medical image processing, machine learning, and GPU parallel computing. 